\title{
The Use of LIDAR Technology in Architectural Offices
}

\author{
Ahmet Murat Erturan ${ }^{1}$, Akif Durdu ${ }^{2}$ and Elif Merve Erturan ${ }^{3}$ \\ ${ }^{1}$ Department of Electrical Electronical Engineering, Konya Technical University. \\ ${ }^{2}$ Department of Electrical Electronical Engineering, Konya Technical University. \\ ${ }^{3}$ Department of Architecture, Konya Technical University.
}

\section{ARTICLE INFO}

\section{Keywords:}

SLAM; interior

architecture; LIDAR

measurement in

architecture; LIDAR

technology; new

technologies in

architecture

\begin{abstract}
Together with the developing technology, robotic systems are used in many areas such as defence technologies, manufacturing industry, search and rescue. The integration of these systems into different areas decreases the need for manpower and has a positive effect on cost. From the past to the present, architectural structures have been made by man-power. On the other hand, the effects of robotics systems in the construction have been observed in recent years. Especially with the usage of 3-dimensional printers and building materials, the construction time is shortened and large financial gains have begun. The use of technology is also seen in the field of interior architecture. By means of machine learning and deep learning algorithms, different furniture and home decorations have been selected according to the dimensions of the room. For example, the algorithm chooses the highest accuracy to have the best visualization and usage area of the furniture to be selected considering the room size and shape. In this paper, it is aimed to map the interior decoration environment through the LIDAR. It is clear that it is difficult to take measurements and prepare plans with traditional methods. The LIDAR, an advanced laser distance measuring sensor, measures the distance as a result of returning the beam spread by itself. The time of LIDAR measurement can be as short as 1 minute. Although it is not a millimeter, it is possible to map draft plans and space sizes in a very short time. Using the Hector Mapping algorithm, a house mapped with LIDAR was found to give high results in terms of accuracy, while it was found to be a great gain in terms of time. The house was fully mapped and the reference door width was entered and the place was made ready for interior decoration.
\end{abstract}

$\square$ Corresponding Author E-Mail Address: elifmerveyilmaz91@hotmail.com 


\section{Introduction}

Nowadays, with the developing technology, the need for manpower has decreased and the machines have been able to do almost anything. In this way, time is also achieved and error rates have become quite low. Architecture is also seen as a space where the need for the architect is reduced. The fact that artificial intelligence can make architectural design has opened up different fields of application especially in the field of interior architecture. Now, mobile applications can be downloaded and we can easily design a space. However, when architects want to redesign the design of a space, they must first take the dimensions of the space in detail. After drawing out the draft plans, they clear these plans and then make drawings on the computer. In this article, it is investigated how a long process can be done in a faster and more practical way in order to facilitate the work of architects.

On the other hand, simultaneous localization and mapping is an important problem for autonomous mobile robots. Mapping a closed environment requires the system to be positioned at the same time. Positioning in open environments is ensured by GPS and reliable results are obtained. In closed environments, reliable results cannot be obtained since GPS signals cannot be received in sufficient amounts. Several methods have been used to solve this problem. Positioning can be made by many methods such as Odometry, Iterative Closest Point, Adaptive Monte Carlo Localization or EKF filters methods [1-2]. Working with 3-D laser scanning technique, the rays coming out of the tower hit the obstacle periodically and go back. As the speed of light and the turnaround time of the beam are known, the distance between the LIDAR and the obstacle is easily determined. Through this beam sent in the form of a point cloud, each point of the obstacle is detected and the obstacle distance is determined. LIDAR is preferred for mapping operations due to the ease of measurement of the distance to LIDAR in closed environments. There are also studies using various sensors such as RGB-D, Sonar, Radar, but LIDAR is preferred for reliability [3].

An autonomous robot is mapping the environment in an enclosed environment and positioning itself simultaneously in this environment is called SLAM (Simultaneous Localization and Mapping). The parametrical methods, such as Kalman Filter and Extended Kalman Filter, have been used for the last 25 years on the SLAM problem. In addition to parametric approaches, the Particle Filter approach, which was introduced for the first time in 2009, has been particularly advantageous in terms of high accuracy and rapid convergence [4]. Particle Filter, which is not connected to a parametrical method such as Gaussian Distribution, is more accurate and faster than other estimation filters.

Several studies have been conducted on mapping of closed environments. Meter or laser meter methods are used for mapping or planning the environment with traditional methods. For the measurement of a room of a dwelling, lengths are determined by measuring from one wall to another wall by meter or laser meter. However, this process is a process that can bring a long and error rate. Because if the ground is not measured in parallel, the measurement results will be inaccurate. In addition, curves, columns or protrusions in the room make measurement difficult and reduce accuracy. 
For the implementation of evolving documentation technologies (digital photography, LIDAR, Laser Scanning and digital photogrammetry), institutional support is also needed to obtain metric data.[12]

\section{SLAM and Used Sensors}

\subsection{SLAM}

It is called SLAM to map the unknown position of autonomous moving robots in an unknown environment to the environment by locating the sensors and positioning itself in this environment. It is a more difficult algorithm than just positioning algorithms. The positioning of the self is predetermined because it moves in an environment where the robot does not know. The positioning of the robot in the environment depends on the full map. The correct map works in dependency by providing correct positioning [4]. In the probabilistic framework;

$$
p\left(x_{t}, m \mid z_{1: t}, u_{1: t}\right)
$$

Where $x_{t}$; the pose at time $\mathrm{t}$ is the m map, the $z_{1: t}$ measurement matrix $u_{1: t}$ control matrix.

In SLAM applications, many methods such as Kalman Filter [5] or Particle Filter, FastSLAM algorithms [6] have been used. In a study in which SLAM was applied in closed environments such as the tunnel, the tunnel was mapped with LIDAR and the robot positioned itself in this environment [7]. Multi-sensor fusion methods using LIDAR and Sonar sensors have been used to detect transparent objects such as glass when performing SLAM [8]. Due to the inability to receive GPS data in closed environments, correct positioning cannot be performed. Autonomous moving robots take this positioning from the odometer, which provides wheel information [9]. Using the extruded Kalman Filter (EKF) method, SLAM study was performed with LIDAR and MEMS sensor fusion and WLAN sensor fusion was applied to obtain efficient results.

SLAM; It is the method commonly used in the mapping and positioning of autonomous robot robots. It is applied in wheel balanced robots by taking the data of the LIDAR and wheel step which provides the odometry data. Multiple sensor data fusion techniques provide more accurate and reliable results.

\subsection{LIDAR}

One of the sensors used for media mapping and positioning is the LIDAR. The working principle of the LIDAR sensors is based on the principle that a beam of light sends a object of its origin as a result of hitting any object and returning.

$$
\text { Distance }=c T / 2
$$


The RpLIDAR scanner collects data from a range of 6-7 meters with a 360 degree full lap scan. In a 2-D plane, the point cloud collects data from the beam beam. It has a scan speed of $5.5 \mathrm{~Hz}$ and $10 \mathrm{~Hz}$. This LIDAR type, which uses a laser triangular measurement method, achieves high accuracy in closed environments [10].

\section{Implementation}

The application is made in a house $160 \mathrm{~m} 2$ in real time. 4 rooms, 1 kitchen, 2 bathrooms and 1 toilet were drawn on a scale close to the actual plan. Firstly, measurement was done with tape measure and laser distance meter. Later, walls and distances were detected and mapped in LIDAR scanned environment and the map was transferred to Autocad.

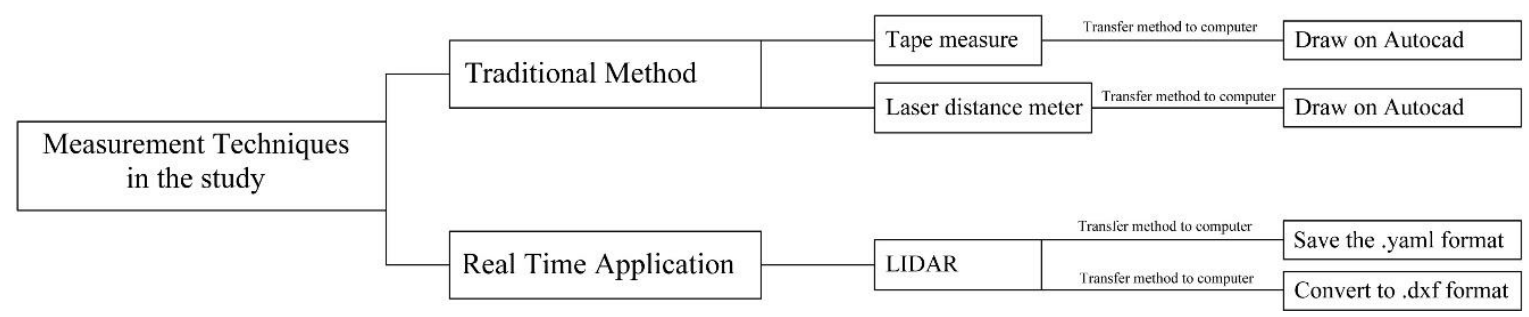

Fig.1. Method of the study

In this study, 3 different measurement tools were tried to be compared. In addition, the plan of drawing the house and transferring it to the computer used in the architectural offices of the Autocad drawing program was used. In order to transfer the plan drawn by LIDAR to Autocad, two different ways were followed. (Fig. 1) The measurement tools used in the study are shown in Fig. 2. In the field of architecture, it is thought that this method, which is used only in the documentation of cultural heritage, will be more popular in time.
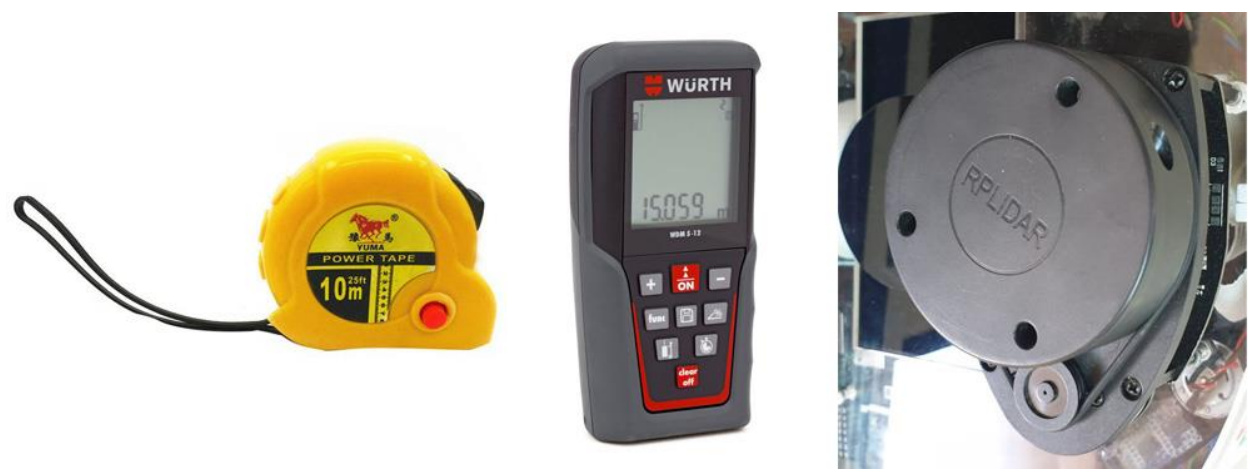

Fig.2. Measurement tools used in the study 


\subsection{Traditional Method}

Using tape measure is one of the traditional measurement techniques in architecture. It is the method of measuring the distances between walls, columns, beams, etc. one by one. This method requires at least two people. Another technique developed at the beginning of the 21st century is the process of making measurements with laser distance meters. In this method, it is sufficient to measure the distance from a single person to a beam from one side to the other. But, both methods

However, in both methods, the measurement person should draw sketches, and write the measurements regularly and verify the measurements on the drafts. Sketches made in Figure 3 show only a part of drafts.
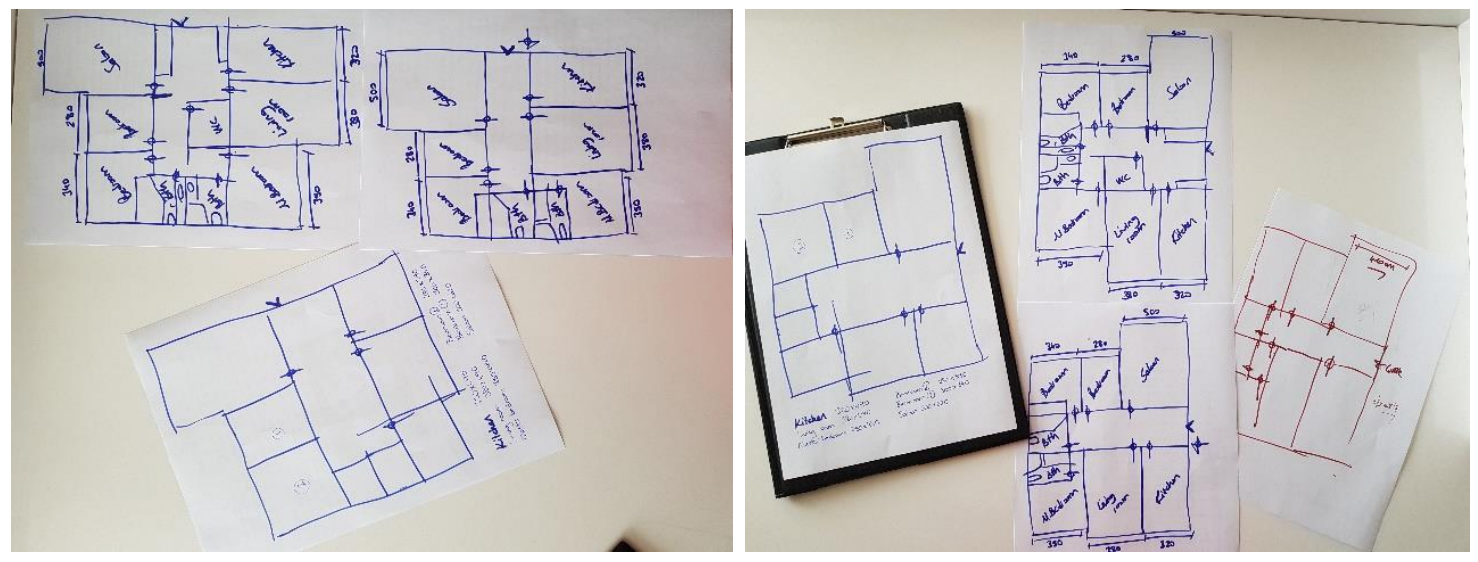

Fig. 3. Sketches 


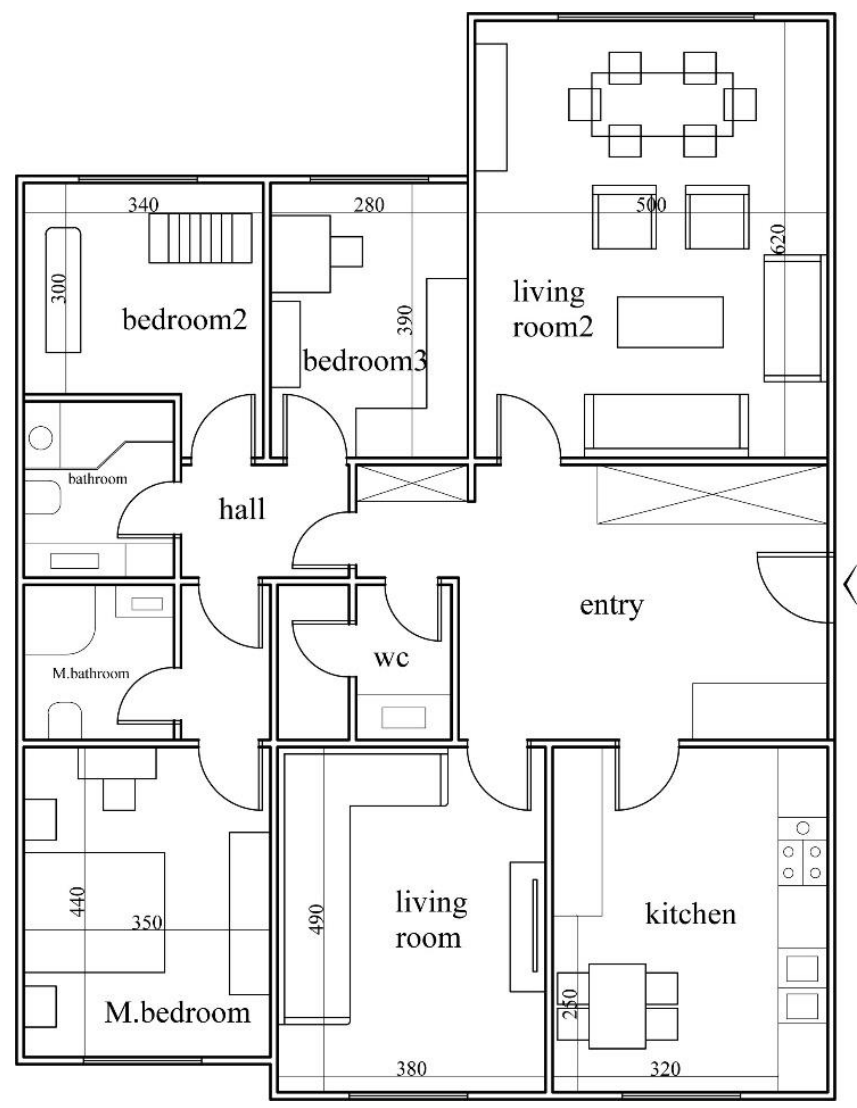

Fig. 4. The house plan

\subsection{Real Time Application with LIDAR}

The method proposed in the article is the measurement technique with LIDAR, which has not yet been applied in the field of architecture. In a very short time, it was determined that he could draw draft plans by sending point clouds to the boundaries in the space. Moreover, it can be transferred to Autocad, which is a frequently used drawing program by architects, is also very easy.

LIDAR scanning was done by computer via Robot Operating System (ROS). Thanks to its robotic application and open source, ROS is an operating system which is used in many areas, especially robotic applications. Traditional SLAM algorithms are based on Bayesian filters and derivatives [11]. Although these algorithms provide stable estimations in small-scale environments, they are unstable due to the accumulation of errors in large-scale environments. Therefore, algorithms such as Rao-Blackwellization and FastSLAM based on Particle Filter are derived. These algorithms are used with the Gmapping package via ROS and are used in SLAM applications together with odometry data. With LIDAR and Particle Filter based HectorSlam 
package, media mapping is produced by end-to-end beam tracking methods without being dependent on odometry data.

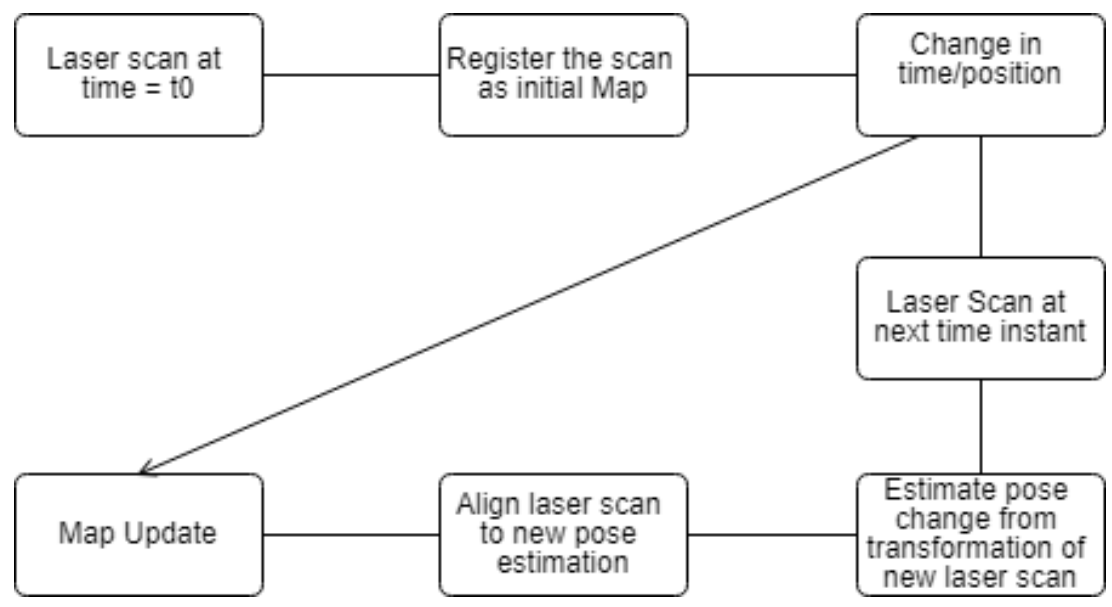

Fig.5. General Flow Diagram

The result of the measurement with LIDAR is shown in Fig.6.

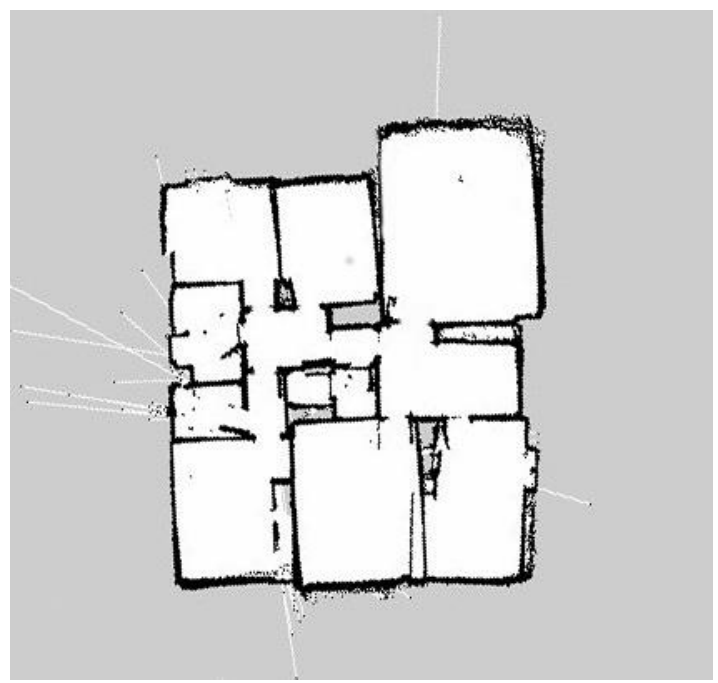

Fig.6. Mapping with LIDAR

In the study, reference was accepted as the width of the door. Considering that the standard door gap is $80 \mathrm{~cm}$, other dimensions of the plans in the Autocad program have also emerged automatically. In Figure 7, the map generated by LIDAR is transferred to Autocad. First of all, measurements were made using traditional methods. Then, the wall lengths of the rooms were measured and the total size of the house was calculated in square meters. In the Autocad, other wall sizes were determined by reference to door width $80 \mathrm{~cm}$. In Fig. 8, kitchen wall length was 
measured with reference to standard $80 \mathrm{~cm}$ kitchen door. Kitchen wall length determined as 490 $\mathrm{cm}$ with traditional measurements (Fig.5); With the LIDAR measurement the door was taken as a reference and was found $510 \mathrm{~cm}$. This method was done for all house walls and the measurements were determined and $95.7 \%$ accuracy was obtained. It was found that the margin of error was at an acceptable level compared to the results obtained with in-situ measurement. The drawings transferred to the Autocad in two ways are shown in Fig. 7.

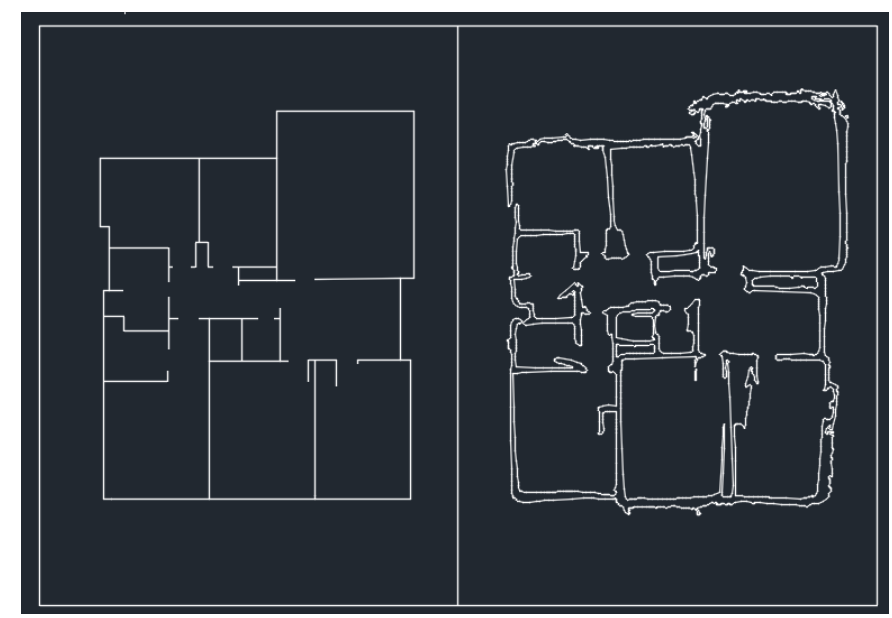

Fig.7. Autocad screen

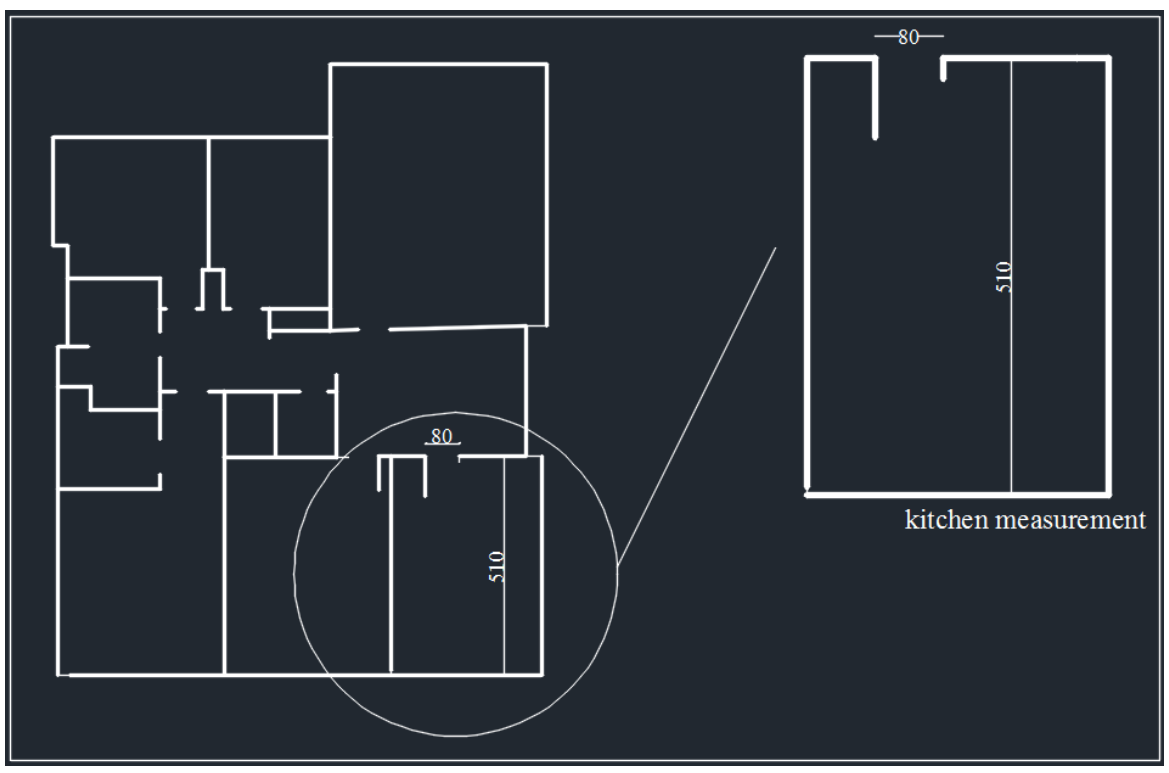

Fig.8.Kitchen Measurements 


\section{Results}

The application consists of two parts, as seen in section 3, and the results were compared. The advantages of technology are once again understood by comparing the traditional measurement techniques and the measurement and measurement times of LIDAR.

LIDAR usage is supported in architecture offices and it is emphasized that both time and labor can be gained. There may be occasional errors in human measurement and it may be necessary to go back and take measurements again. However, it takes seconds to take the measurement again with the LIDAR. Although it is slightly different in terms of cost, it is determined that it will give more accurate results if it is preferred due to measurement accuracy. In addition, since the interior of the house is furnished, especially in high cabinets, it is difficult to measure with laser and meter.

It is necessary to be careful in human measurements and to check the measurements several times. There can be overlooked places. It also becomes difficult to work in indented areas. The human is tired, but the LIDAR is tireless.

\begin{tabular}{|l|l|l|l|}
\hline & Tape measure & $\begin{array}{l}\text { Laser Distance } \\
\text { Meter }\end{array}$ & LIDAR \\
\hline Time & $50-55$ minutes & $40-45$ minutes & $2-3$ minutes \\
\hline $\begin{array}{l}\text { Software-Process } \\
\text { system }\end{array}$ & Autocad & Autocad & Rviz \\
\hline Measuring people & min 3 & $\min 2$ & $0-1$ \\
\hline Accuracy rate & $95-98 \%$ & $95-98 \%$ & $90-95 \%$ \\
\hline
\end{tabular}

Table 1. Comparative analysis

In the study, measurements were made at the house but it is also possible to work on larger scales. It is aimed to make autonomous measurements independent of human in future studies and to calculate these measurements easily by considering the reference values through a software.

\section{References}

1. Xiong, J., Liu, Y., Ye, X., Han, L., Qian, H., Xu, Y.: 'A Hybrid LIDAR-based Indoor Navigation System Enhanced by Ceiling Visual Codes for Mobile Robots', Proceedings of the 2016 IEEE International Conference on Robotics and Biomimetics Qingdao, 2016

2. Nazemzadeh, P., Macii, D.: 'Indoor Localization of Mobile Robots Through QR Code Detection and Dead Reckoning Data Fusion', IEEE/ASME Transactions on Mechatronics, Vol. 22, No. 6, 2017

3. Bassiri, A., Oskoei, M. A., Basiri, A., 'Particle Filter and Finite Impulse Response Filter Fusion and Hector SLAM to Improve the Performance of Robot Positioning', Hindawi Journal of Robotics Volume Article ID 7806854, 2018 
4. Duymaz, E., Oğuz, A. E., Temeltaş, H., 'Eş zamanlı konum belirleme ve haritalama probleminde yeni bir durum tahmin yöntemi olarak parçacık akış filtresi', Journal of the Faculty of Engineering and Architecture of Gazi University 32:4, 2017

5. Barrau, A., Bonnabel, S., An EKF-SLAM algorithm with consistency properties, 2016

6. Wang, H., Wei, S., Chen, Y., 'An Improved Rao-Blackwellized Particle filter for SLAM', International Symposium on Intelligent Information Technology Application Workshops

7. Rubinstein, A., Erez, T., 'Autonomous Robot for Tunnel Mapping', ISCEE International Conference on the Science of Electrical Engineering, 2016

8. Zhong, T., Chong, Z. J., Fu, J. G. M., Pendleton, S., Ang Jr, M. H., 'Sensor Fusion for Localization, Mapping and Navigation in an Indoor Environment', 7th IEEE International Conference Humanoid, Nanotechnology, Information Technology Communication and Control, Environment and Management (HNICEM), 2014

9. Atia, M. M., Liu, S., Nematallah, H., Karamat, T. B., Noureldin, A., 'Integrated Indoor Navigation System for Ground Vehicles With Automatic 3-D Alignment and Position Initialization', IEEE Transactions on Vehicular Technology, Vol. 64, No. 4, 2015

10. Korkmaz M., Durdu, A., Tusun, Y. E., 'Sensor Comparison for a Real-Time SLAM Application', International Journal of Information and Electronics Engineering, Vol. 8, No. 1,2018

11. Funchun, L., Yifeng, C., Yunze, L., 'Research on Indoor Robot SLAM of RBPF Improved with Geometrical Characteristic Localization', 29th Chinese Control And Decision Conference (CCDC), 2017

12. Güleç Korumaz A., Dülgerler O. N., Yakar M.,,'Kültürel Mirasin Belgelenmesinde Dijital Yaklaşimlar', J. Fac.Eng.Arch. Selcuk Univ., v.26, n.3, 2011 